\title{
Discourse Anaphora
}

\author{
Joke Dorrepaal \\ OTS RUU Utrecht \\ dorrepaal@hutruu59.BI'Tnet
}

\section{Abstract}

This paper reports on a model that serves anaphora resolution. A distinction will be made between possible antecedents and preforred antecedents. The set of linguistically possible candidates will be defined in terms of compatibility and recency. Preferred antecedents are a subset of the possible antecedents, selected by the application of extralinguistic knowledge. Motivation for the particular design and comparison with other approaches are extensive.

\section{Introduction}

Both in formal semantics and in NLP, the issue of (pro)nominal reference has aroused much interest. Formal discourse semantics was mainly inspired by the Discourse Representation Theory developed by Kamp (1981) and, the closely related, File Change Semantics (Heim, 1982). In standard DR'T, hardly any restrictions were imposed on the relation between antecedent and anaphor.

This contrasts strongly with work done in the NLP area. Knowledge of the world and intricate inferences play key roles in restricting the number of antecedents for anaphora. Insights from artificial intelligence, linguistics and psychology are all integrated into complex procedures.

The model I will describe jn this paper considers the linguistic context as a means to restrict the large number of interpretations that an expres- sion in principle has to a limited number of alternatives. Knowledge of the world and/or prefercnce of one alternative to the others may only restrict the domain further, never stretch it. This view will be defended in the paper. The former limitation is discussed under the name of 'possible' analyses, the latter is christened 'preferred' analyses.

I will first introduce the model in the next section. Sections 4 ard 5 will motivate the approach and compare it to other proposals. Section 6 will describe and discuss the so-called associative anaphora and show that the model adopted can be extended to non-standard anaphoric reference as well.

\section{The Modell}

The model makes a distinction between possible and preferred antecedents for a particular anaphor. The wellformedness definition below expresses the relation between an anaphor and its possible antecedents. Key notions in the definition are compatibility and recency.

Definition 1 An antecedent Ant is a possible antecedent for an anaphor Ana iff

- Ant and Ana are compatible

- no antecedent compatible with Ana occurs in a more recent unit

The units referred to in the definition relate to units in the discourse. The data structure re- 


\begin{tabular}{|c|c|c|c|c|c|c|}
\hline chap.1 & chap.2 & par. 1 & par.2 & $\mathrm{s} 1$ & $\mathrm{~s} 2$ & s3 \\
\hline
\end{tabular}

Figure 1: The linguistic context

flects the structure of the text, be it that only current units show internal structure. Figure 1 pictures the state of affairs at the time the 4 th sentence in chapter 3, paragraph 3 of a book is being processed. The current chapter, paragraph and sentence show substructure, the others have no internal structure at all. ${ }^{1}$

Recency and compatibility are the central notions in the definition. The notion of recency needs no further explanation. Its importance for anaphora resolution has been demonstrated convincingly by Hobbs (1978). Compatibility demands agreement of linguistic features relevant to anaphora resolution. Pronoun resolution signals the need for features such as category, person, number, gender and humanness. Definite NP resolution requires a more sophisticated mechanism. It should also be able to recognize relations such as synonymy, hyperonymy etc.

Besides the wellformedness definition, one might want to adopt preference rules to select a (small set of) preferred antecedent(s) from the set of possible ones. I will not particularly be concerned with the preference rules in this paper, though 1 do foresee two areas that will determine the nature of these rules. The first is world knowledge. In some cases, only extremely complex inferences can decide between two linguistically equally preferred antecedents. The second is anaphoric preference. An anaphor sometimes prefers an object to a subject or a pronoun to a full NP, everything else being equal. "The truth is

\footnotetext{
${ }^{1}$ The model unintendedly suggests that the units are syntactic in nature and in linear order. A more realistic picture includes semantic units and substructures, as argued for by a.o. Grosz(1977), Scha et.al.(1988)
}

that everything else seldom is equal. Preference is very subtle and influenced by factors like sublanguage, style etc. The discussion to follow will mainly be concerned with possible antecedents.

\section{Motivation}

The approach claims that anaphoric resolution is a two-stage procedure. The first stage determines the possible antecedents by means of the linguistically defined notions of compatibility and recency. The second stage consists of the calculation of preferred antecedents. I will first argue why the distinction between possible and preferred is important. And second, I will motivate why the definition of possible antecedents is expressed in terms of the notions compatibility and recency.

The distinction possible vs. preferred is considered essential for the following reasons. First, it is a means to determine whether a discourse is coherent or not, from the anaphoric point of view. Consider what happens when an antecedent is selected but needs to be rejected because of information later in the discourse. As the meanings of terms like possible/impossible suggest, there is no way that we can backtrack on an initially possible antecedent in favour of an antecedent that is not part of the set of possible ones. Compare the following two discourses:

(1) John was late for his appointment with Joe Fortunately, Joe was even later His work had kept him from leaving in time He, on the other hand, had missed the bus 
(2) John was late for his appointment with Mary

Fortunately, Mary was even later

His work had kept him from leaving in time She, on the other hand, had missed the bus

Discourse (1) is clearly incoherent whereas (2) is acceptable. The reasons are purely linguistic. The definition of possible antecedents above stated that some antecedents are not accessible to a pronoun because of other intermediate compatible antecedents. Only the latter are possible candidates. When the continuation of the discourse makes clear that they were not the right antecedents after all, the discourse should be considered incoherent. A preferred antecedent, however, may be rejected later on in the discourse in favour of one that was not preferred, merely possible. An algorithm that merges possible and preferred is not able to make this distinction.

The second motivation for maintaining the possible/preferred distinction is provided by (linguistic) ambiguity in the language. Consider (3) and the examples cited in Winograd(1972), (4) vs. (5).

(3) The women met their husbands at a party They were very young at the time

(4) The city councillors refused the demonstrators a permit because they feared violence

(5) The city councillors refused the demonstrators a permit because they advocated violence

Discourse (3) is three-way ambiguous and it is vague which of the meanings is intended. Blurring the distinction between preferred and possible antecedents right away contradicts the intuition that all three antecedents in the first sentence might serve as antecedents for the pronoun but not any NP that was mentioned before this utterance. The examples (4) and (5) serve to show that linguistically possible is essentially different from possible as regards 'the world'. Assuming that no NLP-system, nor any human discourse participant by the way, has rich enough information to infer all essentials at the right time (yet), this approach at least yields a reliable, and finite, set of possible candidates.

Having shown that the distinction between wellformed and preferred antecedent-anaphor relations is necessary, it remains to be shown why possible antecedents are defined by means of the simple notions of compatibility and recency. The explanation is quite simple: they seem to yield the right results. It is well-known that full definite NPs are in general further apart from their antecedents than pronouns are. Now consider that definite NPs, in general again, have more descriptive content than pronouns do. It follows that pronouns may be compatible with antecedents that definite NPs do not match with. Together with the notion of recency, compatibility then explains why the antecedents of definite NPs may be at quite a distance. This only holds for definite NPs that have ample descriptive content though. An underspecified definite NP like 'the man' behaves similarly to the pronoun 'he'. Both will accept the most recently mentioned male individual(s) as their possible antecedent(s). So, the number of units that the anaphor may search to find its antecedent is $\mathrm{dy}$ namic rather than static. Consider discourse (1) versus (2) again. A static number of sentences would not explain the difference. The pronouns in (1) behave similar to those in (2) and yet, one of the two is incoherent. The reason is that 'John' in (1) is not accessible because of the intervening NP 'Joe'. 'The NP 'Mary' in (2) does not block reference to 'John' because 'Mary' is not compatible with the same anaphor.

\section{Comparison to Other Ap- proaches}

It might be argued that the notion of possible antecedents is hardly original in the literature on 
the topic of anaphora resolution. The Focusing Approach reported on in Sidner (1983) also employs a list that contains a number of antecedents from which the anaphor can select its co-referent. (cf. also Brennan et.al. 1987, Grosz 1983). Note, however, that the members on this list are substantially different from the possible antecedents described in this paper. Potential antecedents in the Focusing Approach are not determined by the descriptive content of the anaphor but rather by what the discourse makes available for further reference. Suppose a sentence yields 'the pump' and 'the men' as available antecedents. They will be on the list from which a subsequent pronoun, say 'they', selects its antecedent. The difference is that 'the pump' may be potential in the Focusing Approach but in no way "possible' in the sense I used the term. Moreover, all potential antecedents in the Focusing Approach may be rejected in favour of an antecedent selected by a more global mechanism. This means that even the function of Sidner's list of potential antecedents in the discourse model differs from mine. I claim that failure in finding a co-referent on the list of possible antecedents corresponds to incoherence of the discourse. The list employed in the Focusing Approach has no such function. The anaphor could still find a referent on the more global part of the data structure, the socalled Focus Stack.

\section{Associative Anaphora}

To illustrate what the model has in store for a non-standard type of anaphoric reference, consider a case of the 'associative anaphoric use', as discussed in Hawkins(1978). A typical example of the phenomenon is in (6).

(6) The man drove by in a car. The exhaust fumes were terrible. (cf. its exhaust fumes)

Let us see how these constructions behave w.r.t. the model presented above. In view of the close correspondence between the bare associative anaphor and an NP that includes the an- tecedent in pronominal form, I propose to analyse the constructions as having an empty pronoun. The pronoun is anaphoric. Given the notion of compatibility and the fact that empty pronouns have no descriptive content at all, they are predicted to be compatible with any NP. Combining it with the notion of recency, it means that the set of possible antecedents consists of all and only the NPs in the most recent unit. To see whether this is correct, compare the following examples:

(7) I drove by our house in my car The windows were dirty

(8) I drove by our house in my car I saw my father's car The windows were dirty

(9) I drove by our house in my car I saw my father's bicycle The windows were dirty

(10) I drove by our house in my car The windows were dirty The front door was open

In (7), both 'our house' and 'my car' are possible antecedents for the associative anaphor in the second sentence of the discourse. In (8) however, only 'my father's car' is possibly coreferent with the empty pronoun. This indeed is what the model presented above predicts. Intervening potential antecedents block coreference with previous candidates. The NP 'my father's car' is compatible and in a more recent unit than 'our house' as well as 'my car'. Remember that the antecedents we are talking about are possible antecedents in the sense of definition 1 above. World knowledge or subsequent information in the discourse might still consider them inappropriate. This is illustrated in (9). It is 'my father's bicycle' that is the only possible antecedent. Ruling it out by world knowledge and having no possible alternative renders the discourse incoherent. 
Discourse (10) might seem to be a counterexample since 'the front door' can refer to 'the house' despite the occurence of 'the windows' in between. However, 'the windows' was not the only antecedent in the most recent unit. Remember that we've assumed an empty pronoun all along. Among the most recent compatible antecedents is the empty pronoun that corefers to 'house'. This explains why you can have chains of associative anaphora that superficially scem to corefer with an antecedent in a unit preceding the one in which the most recent compatible antecedent occurs.

\section{Conclusion}

The paper reported on a model that serves anaphora resolution. It might be characterized as a two-stage model. The first stage determined the set of linguistically possible antecedents. It was argued that compatibility and recency are adequate notions in determining the set of possible antecedents. The task of the second stage is to restrict the set of possible antecedents to (a number of) preferred antecedent(s).

\section{Acknowledgments}

Louis des Tombe, thanks for your helpful suggestions and critical remarks. I was supported by the European Community and the NBBI through the Eurotra project.

\section{References}

[1] S. R. Brennan, M. W. Friedman, and C. J. Pollard. A centering approach to pronouns. In Proceedings of the 25th Annual Mecting of the Association for Computational Linguislics, 1987.

[2] B. Grosz. The Representation and Use of Fo cus in a System for Understanding Dialogues. In Proceedings of the fifth International Joint
Conference on Artificial Intelligence, Cambridge, Massachusetts, 1977.

[3] J. Mawkins. Definiteness and Indefiniteness. London: Croom Helm, 1978.

[4] I. Heim. The Semantics of Definite and $\mathrm{In}$ definite Noun Phrases. Unpublished PhD dissertation, University of Massachusetts, Amherst, 1982.

[5] J. Mobbs. Resolving pronoun references. Lingua, 44, 1978.

[6] II. Kamp A theory of truth and semantic interpretation. In J. Groenendijk et.al. (eds.) Formal Methods in the Study of Language. Amsterdam: Mathematical Centre, 1981.

[7] R. Scha and L. Polanyi. An Augmented Contex Free Grammar for Discourse. In Procecdings of Coling Budapest, 1988.

[8] C. I. Sidner. Focusing in the comprehension of definite anaphora. In M. Brady and R. C. Berwick (eds.), Computational Models of Discourse. MIT press, 1983.

[9] T. Winograd. Understanding Natural Language. New York: Academic Press, 1972. 